\title{
A generalized significance testing method for global measures of spatial association: an extension of the Mantel test
}

\author{
Sang-Il Lee \\ Department of Geography Education, Seoul National University, San 56-1, Sillim-Dong, \\ Gwanak-Gu, Seoul 151-742, South Korea; e-mail: si_lee@snu.ac.kr \\ Received 13 July 2001; in revised form 26 December 2003
}

\begin{abstract}
This research is concerned with providing a generalized significance testing method for global measures of spatial association by extending the Mantel test. Even though it has long been recognized that univariate spatial association measures such as Moran's $I$ and Geary's $c$ are special cases of Mantel's generalized association statistic, an intensive and comprehensive examination of the connections, particularly in terms of significance testing has never been undertaken. Furthermore, researchers have faced difficulties in dealing with spatial weights matrices with nonzero diagonal elements, and establishing the significance testing method for bivariate spatial association measures such as Cross-Moran and Lee's $L$. The author demonstrates that the proposed extended Mantel test can be applied to any global measure of spatial association with any form of spatial weights matrix in order to approximate the first two moments of the measures. A Monte Carlo simulation for each measure with various forms of spatial weights matrices confirms the exactness of the approximation.
\end{abstract}

\section{Introduction}

The development and elaboration of spatial association measures have recently been focal points in a wide range of disciplines dealing with geographically referenced data. In univariate situations, spatial autocorrelation indices, such as Moran's I and Geary's $c$ have been intensively utilized to parameterize univariate spatial dependence (Cliff and Ord, 1981; Goodchild, 1986; Griffith, 1987; Odland, 1988). In bivariate situations, Cross - Moran has been formulated (Wartenberg, 1985) and illustrated (Griffith, 1993; 1995; Griffith and Layne, 1999). Hubert and his associates developed a nonparametric bivariate spatial association measure (Hubert and Golledge, 1982; Hubert et al, 1985). Finally, Lee (2001a) proposed a parametric bivariate spatial association measure $(L)$ for gauging bivariate spatial dependence by integrating Pearson's correlation coefficient and Moran's $I$.

For a measure to be confirmatory, a significance testing method should be provided. With respect to this, hypothesis testing for univariate spatial association measures is well established: distributional moments of Moran's $I$ and Geary's $c$ have been approximated by sets of equations predicated on both the normality and the randomization assumptions (Cliff and Ord, 1981); exact testing procedures have also been undertaken (Hepple, 1998; Tiefelsdorf, 1998; 2000; Tiefelsdorf and Boots, 1995); and permutation approaches including Monte Carlo simulation have been utilized either as a supplementary or an alternative method (Anselin, 1995; Anselin and Rey, 1991; Kelejian and Robinson, 1998; Sokal et al, 1998). Among these methods, normal approximation with the first two moments, whether based on normality or randomization assumptions, has been preferred not only because it is computationally simple, but also because it provides a reasonable estimation with a sufficient number of observations (Boots and Tiefelsdorf, 2000).

It is recognized that both Moran's I and Geary's $c$ are special cases of Mantel's (1967) generalized cross-product association measure (Cliff and Ord, 1981; Getis, 1991;

- Former address: Department of Geography, The Ohio State University, 1036 Derby Hall, 154 North Oval Mall, Columbus, OH 43210-1361, USA. 
Glick, 1979; Hubert, 1978; Hubert et al, 1981; Sokal, 1979), and that the associated significance testing method can be used to obtain distributional properties of spatial autocorrelation indices (Cliff and Ord, 1981; Upton and Fingleton, 1985). In particular, Hubert et al (1981) strongly appreciate the benefits of generality that Mantel's statistic and the associated randomization inference may provide for spatial association. When two matrices in Mantel's statistic are properly defined for Moran's I and Geary's $c$, one can easily find that Mantel's equations for the first two moments [Mantel, 1967; see also Cliff and Ord, 1981, page 23, equations (1.44)-(1.46)] become identical to those for both measures based on the randomization assumption [see Cliff and Ord, 1981, page 21, equations (1.37), (1.39), and (1.42)]. It follows that Mantel's notion of matrix comparison can be extended to bivariate situations, and their distributional properties can be derived accordingly as attempted (Hubert and Golledge, 1982; Hubert et al, 1985).

It needs to be recognized, however, that the link between the Mantel test and spatial association measures is sustained only when an arbitrary restriction is imposed on defining a spatial weights matrix; that is, diagonal elements are set to zero. This restriction is by no means a universal principle. The importance of considering nonzero diagonals is obvious both for spatial data analysis in general and for spatial association in particular. In a general sense, various forms of map smoothing and cluster detection methods exhaustively utilize the nonzero diagonal scheme for constructing a spatial weights matrix (Haining, 2003, page 85). Also, most spatial weighting schemes based on interobject distances, such as kernel functions, allow a data point itself to have a nonzero value as can be seen in the calibration of geographically weighted regression (Fotheringham et al, 2002, page 56) and the associated summary statistics (Brunsdon et al, 2002).

In the particular context of spatial association, it is worth noting that there are two different perspectives on how to measure spatial dependence: one comparing a reference area with its neighbors; the other regarding a reference area plus its neighbors as a focal set. Evidently, Moran's $I$ and Geary's $c$ belong to the first perspective. However, a global counterpart of a modified Getis - Ord $G_{i}^{*}$ (see Leung et al, 2003, page 730) and Lee's spatial smoothing scalar $S$ (2001a; 2001b) compute a representative value for an overall focal set, which obviously depends upon a nonzero diagonal scheme. This situation is more complicated for bivariate measures. Wartenberg's Cross-Moran compares a data point in one variable and its neighbors in the other variable, mainly because it is just a bivariate extension of Moran's $I$. This substantially erodes the feasibility of the measure in parameterizing the bivariate spatial dependence (Lee, 2001a; Tiefelsdorf, 2001). Hence, it seems inevitable in a bivariate setting that we must compare a focal set in one variable with its counterpart in the other variable by utilizing a nonzero diagonal weighting scheme (Lee, 2001a).

A distinction should be made in discussing the validity of nonzero diagonal schemes between formulating a measure and obtaining its distributional properties. In other words, it is often necessary to transform a matrix with zero diagonal elements to one with nonzero elements to calibrate statistical moments even though the measure does not have to rely on a nonzero scheme in its original formulation. As can be seen throughout the paper, a presentation of Geary's measure in a quadratic form entails a redefinition of the spatial weights matrix, which is necessary to obtain distributional properties of the measure if predicated on the normality assumption or guided by the exact distribution approach. The method presented in this paper will also utilize the transformation.

With respect to this, Heo and Gabriel (1998) succeeded in extending the Mantel test by devising a way of dealing with nonzero diagonal elements in either of two matrices involved. Thus, the main purpose of the present paper is to apply their formulations to global measures of spatial association to obtain an adequate set of distributional properties. Subsequently, I first present an extended Mantel test predicated on Heo and Gabriel 
(1998) by utilizing a set of matrix quantities for the first two moments. Second, I apply the extended Mantel test to five global measures of spatial association; Moran's I, Geary's $c$, the spatial smoothing scalar $S$ (Lee, 2001a; 2001b), Wartenberg's Cross Moran (Wartenberg, 1985), and Lee's L (Lee, 2001a). In doing so, I (1) demonstrate how they are defined according to Mantel's general statistic; (2) provide equations for expected values and variances and discuss variabilities among different forms of spatial weights matrices; (3) compare values approximated by the derived equations with values from 10000 permutations, focusing on the effect of nonzero diagonal elements in a spatial weights matrix; (4) evaluate the applicability of the extended Mantel test to bivariate spatial association measures, specifically to Lee's $L$.

\section{An extended Mantel test}

Mantel's generalized cross-product association measure $(Z)$ was originally proposed to explore spatiotemporal dependence among events (Mantel, 1967), and is given by

$$
Z=\sum_{i} \sum_{j \neq i} x_{i j} y_{i j}=\sum_{i, j \neq i}(\mathbf{X} \circ \mathbf{Y})
$$

where $x_{i j}$ is an element in a spatial (dis)similarity matrix $\mathbf{X}$, and $y_{i j}$ is an element in a temporal (dis)similarity matrix $\mathbf{Y}$, and $\mathbf{X} \circ \mathbf{Y}$ denotes a pairwise dot product between the two matrices. By calculating the sum of pairwise dot products between two matrices, the measure evaluates whether there is a certain relationship between spatial distance and temporal distance between the members of all possible $n(n-1)$ pairs (Mantel, 1967). Based on the randomization assumption, the expected value (E) and variance can be presented as (see Cliff and Ord, 1981, page 23)

$$
\begin{aligned}
\mathrm{E}(Z)= & \frac{S_{0} T_{0}}{n(n-1)}, \\
\operatorname{var}(Z)= & \frac{S_{1} T_{1}}{2 n(n-1)}+\frac{\left(S_{2}-2 S_{1}\right)\left(T_{2}-2 T_{1}\right)}{4 n(n-1)(n-2)} \\
& \quad+\frac{\left(S_{0}^{2}+S_{1}-S_{2}\right)\left(T_{0}^{2}+T_{1}-T_{2}\right)}{n(n-1)(n-2)(n-3)}-[\mathrm{E}(Z)]^{2},
\end{aligned}
$$

where

$$
\begin{aligned}
& S_{0}=\sum_{(2)} w_{i j}, \quad T_{0}=\sum_{(2)} t_{i j}, \\
& S_{1}=\frac{1}{2} \sum_{(2)}\left(w_{i j}+w_{j i}\right)^{2},
\end{aligned}
$$

$w_{i j}$ is the weight assigned to a link between spatial objects $i$ and $j$,

$t_{i j}$ is the value assigned to the pair of spatial objects $i$ and $j$,

$$
\begin{aligned}
& w_{i .}=\sum_{j=1}^{n} w_{i j}, \quad w_{. i}=\sum_{j=1}^{n} w_{j i}, \\
& t_{i .}=\sum_{j=1}^{n} t_{i j}, \quad t_{. i}=\sum_{j=1}^{n} t_{j i}, \\
& \sum_{(2)}=\sum_{i=1}^{n} \sum_{\substack{j=1 \\
i \neq j}}^{n} .
\end{aligned}
$$


Note that a restriction of $i \neq j$ is required for all the elements in equations (1) and (2). In other words, diagonal elements in at least one of the two matrices should be set to zero.

By eliminating the restriction, a generalized global measure of spatial association $(\Gamma)$ is defined as

$$
\Gamma=\sum_{i} \sum_{j} p_{i j} q_{i j}=\sum_{i, j}(\mathbf{P} \circ \mathbf{Q})=\operatorname{tr}\left(\mathbf{P}^{\mathrm{T}} \mathbf{Q}\right)=\operatorname{tr}\left(\mathbf{P} \mathbf{Q}^{\mathrm{T}}\right),
$$

where $\mathbf{P}$ is a matrix of spatial proximity of locations, and $\mathbf{Q}$ is a matrix of numeric proximity of values on those locations (Haining, 1990, page 230). The measure is obtained by summing all the pairwise dot products or all the diagonal elements of the inner or outer product of $\mathbf{P}$ and $\mathbf{Q}$. An overall expected value needs to be decomposed into two elements, one for off-diagonal elements and the other for on-diagonal:

$$
\mathrm{E}(\Gamma)=\mathrm{E}\left(\Gamma^{\text {off }}\right)+\mathrm{E}\left(\Gamma^{\text {on }}\right) .
$$

Accordingly, an overall variance is decomposed into three elements (Heo and Gabriel, 1998, page 847).

$$
\operatorname{var}(\Gamma)=\operatorname{var}\left(\Gamma^{\text {off }}\right)+\operatorname{var}\left(\Gamma^{\text {on }}\right)+2 \operatorname{cov}\left(\Gamma^{\text {off }}, \Gamma^{\text {on }}\right) .
$$

The equations that will be presented for all the elements in equations (4) and (5) are based on what Mantel calls a "finite population approach" (1967, page 213). This is basically identical to what has been called the randomization approach. Rows and columns of matrix $\mathbf{Q}$ are permuted while those of the $\mathbf{P}$ matrix are arbitrarily kept the same. Mantel makes a requirement in the permutation process that "if any 2 rows are permuted, the corresponding 2 columns are also permuted so that, for each $i$, the $i$ th row and $i$ th column will correspond to the same case" (1967, page 215).

Although only six quantities are needed to define the original equations [(1) and (2)] (Mantel and Valand, 1970), some additional quantities need to be defined, at least

\begin{tabular}{|c|c|c|c|}
\hline \multicolumn{2}{|l|}{$\mathbf{P}$ matrix } & \multicolumn{2}{|l|}{$\mathbf{Q}$ matrix } \\
\hline quantity & notation & quantity & notation \\
\hline$F_{0}^{\text {off }}$ & $\sum_{i} \sum_{j \neq i} p_{i j}=\mathbf{1}^{\mathrm{T}} \mathbf{P} \mathbf{1}-\operatorname{tr}(\mathbf{P})$ & $G_{0}^{\text {off }}$ & $\begin{array}{l}\text { As for } \mathbf{P} \text { with } \\
p \text { replaced by } q \\
\text { and } \mathbf{P} \text { replaced by } \mathbf{Q}\end{array}$ \\
\hline$F_{0}^{\text {on }}$ & $\sum_{i} p_{i i}=\operatorname{tr}(\mathbf{P})$ & $G_{0}^{\text {on }}$ & \\
\hline$F_{1}^{\text {off }}$ & $\sum_{i} \sum_{j \neq i} p_{i j}^{2}=\operatorname{tr}\left(\mathbf{P}^{\mathrm{T}} \mathbf{P}\right)-\left[\operatorname{diag}(\mathbf{P})^{\mathrm{T}} \operatorname{diag}(\mathbf{P})\right]$ & $G_{1}^{\text {off }}$ & \\
\hline$F_{1}^{\text {on }}$ & $\sum_{i} p_{i i}^{2}=\operatorname{diag}(\mathbf{P})^{\mathrm{T}} \operatorname{diag}(\mathbf{P})$ & $G_{1}^{\text {on }}$ & \\
\hline$F_{2}^{\text {off }}$ & $\sum_{i}\left(\sum_{i \neq i} p_{i j}\right)^{2}=[\mathbf{P} \mathbf{1}-\operatorname{diag}(\mathbf{P})]^{\mathrm{T}}[\mathbf{P} \mathbf{1}-\operatorname{diag}(\mathbf{P})]$ & $G_{2}^{\text {off }}$ & \\
\hline$F_{2}^{\text {all }}$ & $\sum\left(\sum p_{i j}\right)^{2}=(\mathbf{P} \mathbf{1})^{\mathrm{T}}(\mathbf{P} \mathbf{1})=\mathbf{1}^{\mathrm{T}}\left(\mathbf{P}^{\mathrm{T}} \mathbf{P}\right) \boldsymbol{1}$ & $G_{2}^{\text {all }}$ & \\
\hline
\end{tabular}
twelve being necessary in total (table 1), because those original quantities are relevant

Table 1. Twelve quantities of matrices $\mathbf{P}$ and $\mathbf{Q}$.

Note. 1 is an $n \times 1$ vector of ones and $\operatorname{diag}()$ is an operator which yields a column vector consisting of the diagonal elements of a matrix. Note that, without loss of generality, both $\mathbf{P}$ and $\mathbf{Q}$ need to be symmetrized. 
only to off-diagonal elements. One restriction is that both matrices should be symmetric to compute those quantities. An asymmetric matrix (for example, row-standardized spatial weights matrices) can be rendered symmetric by an equation, $\frac{1}{2}\left(\mathbf{P}+\mathbf{P}^{\mathrm{T}}\right)$.

From equations (4) and (5), one can see that five elements need to be obtained in order to compute the expected value and variance; two for the expected value $\left[\mathrm{E}\left(\Gamma^{\mathrm{off}}\right)\right.$ and $\left.\mathrm{E}\left(\Gamma^{\mathrm{on}}\right)\right]$ and three for the variance $\left[\operatorname{var}\left(\Gamma^{\mathrm{off}}\right), \operatorname{var}\left(\Gamma^{\mathrm{on}}\right)\right.$, and $\left.\operatorname{cov}\left(\Gamma^{\mathrm{off}}, \Gamma^{\mathrm{on}}\right)\right]$. Table 2 presents equations for the five elements utilizing quantities defined in table 1 . Original equations for those elements are given in the appendix. By combining the first two rows in table 2 and referring to quantities in table 1, we obtain an equation for an overall expected value in accordance with equation (4):

$$
\mathrm{E}(\Gamma)=\mathrm{E}\left(\Gamma^{\text {off }}\right)+\mathrm{E}\left(\Gamma^{\text {on }}\right)=\frac{\left.\left[\left(\boldsymbol{1}^{\mathrm{T}} \mathbf{P} \boldsymbol{1}\right)-\operatorname{tr}(\mathbf{P})\right]\left[\boldsymbol{1}^{\mathrm{T}} \mathbf{Q} \boldsymbol{1}\right)-\operatorname{tr}(\mathbf{Q})\right]}{n(n-1)}+\frac{\operatorname{tr}(\mathbf{P}) \operatorname{tr}(\mathbf{Q})}{n},
$$

and an overall variance, $\operatorname{var}(\Gamma)$, can be defined according to equation (5). Here three things should be noticed. First, if diagonal elements in either $\mathbf{P}$ or $\mathbf{Q}$ are set to zero, then the overall expected value reduces to one for off-diagonal elements, because $\mathrm{E}\left(\Gamma^{\text {on }}\right)$ will be equal to zero. If a sum of all the elements in either $\mathbf{P}$ or $\mathbf{Q}$ turns out to have a constant value, $\mathrm{E}\left(\Gamma^{\text {off }}\right)$ will be further simplified. Second, equation (2) and the equation for $\operatorname{var}\left(\Gamma^{\text {off }}\right)$ in table 2 that are seemingly different are in fact identical. Third, if there is no on-diagonal variance, the overall variance reduces to the variance for off-diagonal elements alone, because covariance will also be zero.

Table 2. Five elements for the expected value and variance of $\Gamma$ with the quantities defined in table 1 .

Moment Element Notation

\begin{tabular}{|c|c|c|}
\hline \multirow[t]{2}{*}{ Mean } & $\mathrm{E}\left(\Gamma^{\mathrm{off}}\right)$ & $\frac{F_{0}^{\text {off }} G_{0}^{\text {off }}}{n(n-1)}$ \\
\hline & $\mathrm{E}\left(\Gamma^{\mathrm{on}}\right)$ & $\frac{F_{0}^{\text {on }} G_{0}^{\text {off }}}{n}$ \\
\hline \multirow[t]{5}{*}{ Variance } & $\operatorname{var}\left(\Gamma^{\mathrm{off}}\right)$ & $\begin{array}{l}\frac{2 F_{1}^{\text {off }} G_{1}^{\text {off }}}{n(n-1)}+\frac{4\left(F_{2}^{\text {off }}-F_{1}^{\text {off }}\right)\left(G_{2}^{\text {off }}-G_{1}^{\text {off }}\right)}{n(n-1)(n-2)} \\
\quad+\frac{\left[\left(F_{0}^{\text {off }}\right)^{2}+2 F_{1}^{\text {off }}-4 F_{2}^{\text {off }}\right]\left[\left(G_{0}^{\text {off }}\right)^{2}+2 G_{1}^{\text {off }}-4 G_{2}^{\text {off }}\right]}{n(n-1)(n-2)(n-3)}-\left[\mathrm{E}\left(\Gamma^{\text {off }}\right)\right]^{2}\end{array}$ \\
\hline & $\operatorname{var}\left(\Gamma^{\mathrm{on}}\right)$ & $\frac{F_{1}^{\mathrm{on}} G_{1}^{\mathrm{on}}}{n}+\frac{\left[\left(F_{0}^{\mathrm{on}}\right)^{2}-F_{1}^{\mathrm{on}}\right]\left[\left(G_{0}^{\mathrm{on}}\right)^{2}-G_{1}^{\mathrm{on}}\right]}{n(n-1)}-\left[\mathrm{E}\left(\Gamma^{\mathrm{on}}\right)\right]^{2}$ \\
\hline & $\operatorname{cov}\left(\Gamma^{\mathrm{off}}, \Gamma^{\mathrm{on}}\right)$ & $\frac{\left(F_{2}^{\mathrm{all}}-F_{1}^{\mathrm{on}}-F_{2}^{\mathrm{off}}\right)\left(G_{2}^{\mathrm{all}}-G_{1}^{\mathrm{on}}-G_{2}^{\text {off }}\right)}{2 n(n-1)}$ \\
\hline & & $+\frac{\left[\left(F_{0}^{\text {on }} F_{0}^{\text {off }}\right)-\left(F_{2}^{\text {all }}-F_{1}^{\text {on }}-F_{2}^{\text {off }}\right)\right]\left[\left(G_{0}^{\text {on }} G_{0}^{\text {off }}\right)-\left(G_{2}^{\text {all }}-G_{1}^{\text {on }}-G_{2}^{\text {off }}\right)\right]}{n(n-1)(n-2)}$ \\
\hline & & $-\mathrm{E}\left(\Gamma^{\mathrm{off}}\right) \mathrm{E}\left(\Gamma^{\mathrm{on}}\right)$ \\
\hline
\end{tabular}


3 An application of the extended Mantel test to global measures of spatial association Table 3 lists the five global measures of spatial association in both summation and matrix notations. Without loss of generality for spatial weights matrices, $\mathbf{V}$ allows for any method of defining topological relationships among observations. The matrix notation column in the table is presented by utilizing a $z$-transformation procedure [see the annotation in the table and note that a population variance $(\sigma)$ rather than a sample variance is used to compute a $z$-socre].

The matrix notation for Geary's $c$ needs to elaborated. Cliff and Ord (1981, page 167) demonstrate that Geary's $c$ can be presented in a quadratic form like Moran's $I$. Matrix $\boldsymbol{\Omega}$ is defined as a diagonal matrix with nonzero elements, each of which is defined as

$$
\omega_{i i}=\frac{1}{2} \sum_{j}\left(v_{i j}+v_{j i}\right) \quad\left(\text { when } \mathbf{V} \text { is symmetric, } \omega_{i i}=\sum_{j} v_{i j}\right) .
$$

Note that the diagonal of $\mathbf{\Omega}$ becomes identical to a vector of row sums of $\mathbf{V}$, when $\mathbf{V}$ is symmetric.

Table 3. Notation for five global measures of spatial association.

Measure Summation notation

Matrix notation

\section{Univariate}

Moran's I

$$
\frac{n}{\sum_{i} \sum_{j} v_{i j}} \frac{\sum_{i} \sum_{j} v_{i j}\left(x_{i}-\bar{x}\right)\left(x_{j}-\bar{x}\right)}{\sum_{i}\left(x_{i}-\bar{x}\right)^{2}}
$$$$
\frac{\left(\boldsymbol{z}_{X}\right)^{\mathrm{T}} \mathbf{V} \boldsymbol{z}_{X}}{\boldsymbol{1}^{\mathrm{T}} \mathbf{V} \boldsymbol{1}}
$$

Geary's $c$

$$
\frac{n-1}{2 \sum_{i} \sum_{j} v_{i j}} \frac{\sum_{i} \sum_{j} v_{i j}\left(x_{i}-x_{j}\right)^{2}}{\sum_{i}\left(x_{i}-\bar{x}\right)^{2}}
$$

$$
\frac{n-1}{n} \frac{\left(\boldsymbol{z}_{X}\right)^{\mathrm{T}}(\mathbf{\Omega}-\mathbf{V}) \boldsymbol{z}_{X}}{\boldsymbol{1}^{\mathrm{T}} \mathbf{V} \mathbf{1}}
$$

Lee's spatial smoothing scalar $(S)$ (2001a)

$$
\frac{n}{\sum_{i}\left(\sum_{j} v_{i j}\right)^{2}} \frac{\sum_{i}\left(\sum_{j} v_{i j}\left(x_{j}-\bar{x}\right)\right)^{2}}{\sum_{i}\left(x_{i}-\bar{x}\right)^{2}}
$$

\section{Bivariate}

Wartenberg's

Cross-Moran (1985)

$$
\frac{n}{\sum_{i} \sum_{j} v_{i j}} \frac{\sum_{i} \sum_{j} v_{i j}\left(x_{i}-\bar{x}\right)\left(y_{j}-\bar{y}\right)}{\left[\sum_{i}\left(x_{i}-\bar{x}\right)^{2}\right]^{1 / 2}\left[\sum_{i}\left(y_{i}-\bar{y}\right)^{2}\right]^{1 / 2}} \quad \frac{\left(\boldsymbol{z}_{X}\right)^{\mathrm{T}} \mathbf{V} \boldsymbol{z}_{Y}}{\boldsymbol{1}^{\mathrm{T}} \mathbf{V} \mathbf{1}}
$$

Lee's $L$

(2001a)

$$
\frac{n}{\sum_{i}\left(\sum_{j} v_{i j}\right)^{2}} \frac{\sum_{i}\left[\left(\sum_{j} v_{i j}\left(x_{j}-\bar{x}\right)\right)\left(\sum_{j} v_{i j}\left(y_{j}-\bar{y}\right)\right)\right]}{\left[\sum_{i}\left(x_{i}-\bar{x}\right)^{2}\right]^{1 / 2}\left[\sum_{i}\left(y_{i}-\bar{y}\right)^{2}\right]^{1 / 2}} \frac{\left(\boldsymbol{z}_{X}\right)^{\mathrm{T}}\left(\mathbf{V}^{\mathrm{T}} \mathbf{V}\right) \boldsymbol{z}_{Y}}{\boldsymbol{1}^{\mathrm{T}}\left(\mathbf{V}^{\mathrm{T}} \mathbf{V}\right) \boldsymbol{1}}
$$

Note. $\boldsymbol{z}_{X}$ and $\boldsymbol{z}_{Y}$ are $z$-transformed vectors of variables $X$ and $Y$. For example, an element in $\boldsymbol{z}_{X}$ is given by

$$
\left(x_{i}-\bar{x}\right)\left[\sum_{i}\left(x_{i}-\bar{x}\right)^{2} / n\right]^{-1 / 2}=\left(x_{i}-\bar{x}\right) / \sigma_{X} .
$$


The spatial smoothing scalar in table 3 was proposed by Lee (2001a, page 375) and later denoted $S$ (Lee, 2001b), and can be seen as a univariate counterpart of Lee's $L$ just as Moran's $I$ is of Wartenberg's Cross-Moran, which can clearly be seen from the notational equivalence in each pair. The spatial smoothing scalar can also be seen as a global counterpart of a modified version of Getis-Ord's $G_{i}^{*}$ that is derived by just squaring the original equation (Leung et al, 2003, page 730).

By using the equations in the matrix notation, one can define the matrices $\mathbf{P}$ and $\mathbf{Q}$ for each spatial association measure in order to conform to the general form of Mantel's statistic in equation (3) (table 4, over). For all the measures, matrix $\mathbf{P}$ takes the standardized form of a spatial weights matrix. The $\mathbf{P}$ matrix for Geary's $c$ should be further elaborated. For a symmetric $\mathbf{V}$, a matrix $\boldsymbol{\Omega}-\mathbf{V}$ is presented as:

$$
\boldsymbol{\Omega}-\mathbf{V}=\left[\begin{array}{cccc}
\sum_{j} v_{1 j}-v_{11} & -v_{12} & \cdots & -v_{1 n} \\
-v_{21} & \sum_{j} v_{2 j}-v_{22} & \cdots & -v_{2 n} \\
\vdots & \vdots & \ddots & \vdots \\
-v_{n 1} & -v_{n 2} & \cdots & \sum_{j} v_{n j}-v_{n n}
\end{array}\right] .
$$

Because each row sum is equal to zero, the sum of all the elements in the matrix is also equal to zero. On the other hand, the sum of on-diagonal elements is given by

$$
\begin{aligned}
\operatorname{tr}(\mathbf{\Omega}-\mathbf{V}) & =\sum_{i} \sum_{j} v_{i j}-\sum_{i} v_{i i} \\
& =\boldsymbol{1}^{\mathrm{T}} \mathbf{V} \mathbf{1}-\operatorname{tr}(\mathbf{V}) .
\end{aligned}
$$

When equation (7) is applied to the definitional equation for Geary's $c$ in table 4, one can see that the sum of diagonal elements in $\mathbf{P}$ is given by

$$
\operatorname{tr}(\mathbf{P})=\frac{n-1}{n}\left(1-\frac{\operatorname{tr}(\mathbf{V})}{\mathbf{1}^{\mathrm{T}} \mathbf{V} \mathbf{1}}\right)
$$

as seen in the table.

Next, matrix $\mathbf{Q}$ is identical among the univariate measures as well as among the bivariate measures. For the univariate measures, an outer product of a $z$-transformed variable $X$ is presented:

$$
\mathbf{Q}=\left[\begin{array}{cccc}
\frac{\left(x_{1}-\bar{x}\right)\left(x_{1}-\bar{x}\right)}{\sigma_{X}^{2}} & \frac{\left(x_{1}-\bar{x}\right)\left(x_{2}-\bar{x}\right)}{\sigma_{x}^{2}} & \ldots & \frac{\left(x_{1}-\bar{x}\right)\left(x_{n}-\bar{x}\right)}{\sigma_{x}^{2}} \\
\frac{\left(x_{2}-\bar{x}\right)\left(x_{1}-\bar{x}\right)}{\sigma_{X}^{2}} & \frac{\left(x_{2}-\bar{x}\right)\left(x_{2}-\bar{x}\right)}{\sigma_{X}^{2}} & \ldots & \frac{\left(x_{2}-\bar{x}\right)\left(x_{n}-\bar{x}\right)}{\sigma_{X}^{2}} \\
\vdots & \vdots & \ddots & \vdots \\
\frac{\left(x_{n}-\bar{x}\right)\left(x_{1}-\bar{x}\right)}{\sigma_{X}^{2}} & \frac{\left(x_{n}-\bar{x}\right)\left(x_{2}-\bar{x}\right)}{\sigma_{X}^{2}} & \ldots & \frac{\left(x_{n}-\bar{x}\right)\left(x_{n}-\bar{x}\right)}{\sigma_{X}^{2}}
\end{array}\right] .
$$

The sum of off-diagonal elements in an $i$ th row is given by

$$
\begin{aligned}
\sum_{j \neq i} q_{i j} & =\frac{\left(x_{i}-\bar{x}\right)\left[\left(x_{1}-\bar{x}\right)+\ldots+\left(x_{i-1}-\bar{x}\right)+\left(x_{i+1}-\bar{x}\right)+\ldots+\left(x_{n}-\bar{x}\right)\right]}{\sigma_{X}^{2}} \\
& =\frac{\left(x_{i}-\bar{x}\right)\left[\left(\sum_{i=1}^{n}\left(x_{i}-\bar{x}\right)\right)-\left(x_{i}-\bar{x}\right)\right]}{\sigma_{X}^{2}}=\frac{-\left(x_{i}-\bar{x}\right)^{2}}{\sigma_{X}^{2}} .
\end{aligned}
$$


Table 4. Definitions of $\mathbf{P}$ and $\mathbf{Q}$ and the equations for the expected values of five global measures of spatial association.

\begin{tabular}{|c|c|c|c|c|c|c|c|}
\hline \multirow[t]{2}{*}{$\Gamma$} & \multicolumn{3}{|l|}{$\mathbf{P}$} & \multicolumn{3}{|l|}{$\mathbf{Q}$} & \multirow[t]{2}{*}{$\mathrm{E}(\Gamma)$} \\
\hline & definition & $\mathbf{1}^{\mathrm{T}} \mathbf{P} \mathbf{1}$ & $\operatorname{tr}(\mathbf{P})$ & definition & $\mathbf{1}^{\mathrm{T}} \mathbf{Q} \mathbf{1}$ & $\operatorname{tr}(\mathbf{Q})$ & \\
\hline \multicolumn{8}{|l|}{ Univariate } \\
\hline Moran's I & $\frac{\mathrm{V}}{\mathbf{1}^{\mathrm{T}} \mathrm{V} \mathbf{1}}$ & 1 & $\frac{\operatorname{tr}(\mathbf{V})}{\mathbf{1}^{\mathrm{T}} \mathbf{V} \mathbf{1}}$ & $\boldsymbol{z}_{X}\left(\boldsymbol{z}_{X}\right)^{\mathrm{T}}$ & 0 & $n$ & $\left\{n\left[\frac{\operatorname{tr}(\mathbf{V})}{\mathbf{1}^{\mathrm{T}} \mathbf{V} \mathbf{1}}\right]-1\right\} /(n-1)$ \\
\hline Geary's $c$ & $\frac{n-1}{n} \frac{(\boldsymbol{\Omega}-\mathbf{V})}{\boldsymbol{1}^{\mathrm{T}} \mathbf{V} \mathbf{1}}$ & 0 & $\frac{n-1}{n}\left(1-\frac{\operatorname{tr}(\mathbf{V})}{1^{\mathrm{T}} \mathbf{V} \mathbf{1}}\right)$ & & & & $1-\frac{\operatorname{tr}(\mathbf{V})}{\boldsymbol{1}^{\mathrm{T}} \mathbf{V} \mathbf{1}}$ \\
\hline $\begin{array}{l}\text { Lee's spatial } \\
\text { smoothing } \\
\text { scalar }(S)\end{array}$ & $\frac{\mathbf{V}^{\mathrm{T}} \mathbf{V}}{\boldsymbol{1}^{\mathrm{T}}\left(\mathbf{V}^{\mathrm{T}} \mathbf{V}\right) \boldsymbol{l}}$ & 1 & $\frac{\operatorname{tr}\left(\mathbf{V}^{\mathrm{T}} \mathbf{V}\right)}{\boldsymbol{1}^{\mathrm{T}}\left(\mathbf{V}^{\mathrm{T}} \mathbf{V}\right) \boldsymbol{I}}$ & & & & $\left\{n\left[\frac{\operatorname{tr}\left(\mathbf{V}^{\mathrm{T}} \mathbf{V}\right)}{\boldsymbol{1}^{\mathrm{T}}\left(\mathbf{V}^{\mathrm{T}} \mathbf{V}\right) \boldsymbol{I}}\right]-1\right\} /(n-1)$ \\
\hline $\begin{array}{l}\text { Bivariate } \\
\text { Wartenberg's } \\
\text { Cross-Moran }\end{array}$ & $\frac{\mathrm{V}}{\boldsymbol{1}^{\mathrm{T}} \mathrm{V} \mathbf{1}}$ & 1 & $\frac{\operatorname{tr}(\mathbf{V})}{\boldsymbol{1}^{\mathrm{T}} \mathbf{V} \boldsymbol{1}}$ & $\boldsymbol{z}_{X}\left(\boldsymbol{z}_{Y}\right)^{\mathrm{T}}$ & 0 & $n r_{X, Y}$ & $r_{X, Y}\left\{n\left[\frac{\operatorname{tr}(\mathbf{V})}{\boldsymbol{1}^{\mathrm{T}} \mathbf{V} \mathbf{1}}\right]-1\right\} /(n-1)$ \\
\hline Lee's $L$ & $\frac{\mathbf{V}^{\mathrm{T}} \mathbf{V}}{\boldsymbol{1}^{\mathrm{T}}\left(\mathbf{V}^{\mathrm{T}} \mathbf{V}\right) \boldsymbol{1}}$ & 1 & $\frac{\operatorname{tr}\left(\mathbf{V}^{\mathrm{T}} \mathbf{V}\right)}{\boldsymbol{1}^{\mathrm{T}}\left(\mathbf{V}^{\mathrm{T}} \mathbf{V}\right) \boldsymbol{I}}$ & & & & $r_{X, Y}\left\{n\left[\frac{\operatorname{tr}\left(\mathbf{V}^{\mathrm{T}} \mathbf{V}\right)}{\mathbf{1}^{\mathrm{T}}\left(\mathbf{V}^{\mathrm{T}} \mathbf{V}\right) \boldsymbol{1}}\right]-1\right\} /(n-1)$ \\
\hline
\end{tabular}


Therefore, the sum of all the off-diagonal elements in $\mathbf{Q}$ can be computed by summing up all the row sums:

$$
\sum_{i} \sum_{j \neq i} q_{i j}=\frac{-\sum_{i}\left(x_{i}-\bar{x}\right)^{2}}{\sigma_{X}^{2}}=-n .
$$

On the other hand, the sum of on-diagonal elements in equation (8) is given by

$$
\operatorname{tr}(\mathbf{Q})=\sum_{i} q_{i i}=\frac{\sum_{i}\left(x_{i}-\bar{x}\right)^{2}}{\sigma_{X}^{2}}=n .
$$

Thus, the sum of all the elements in $\mathbf{Q}$, that is, $\mathbf{1}^{\mathrm{T}} \mathbf{Q} \mathbf{1}$, will be zero (see table 4 ).

Accordingly, an outer product of $z$-transformed variables $X$ and $Y$ defines the matrix $\mathbf{Q}$ for both Cross - Moran and Lee's $L$, and is given by

$$
\mathbf{Q}=\left[\begin{array}{cccc}
\frac{\left(x_{1}-\bar{x}\right)\left(y_{1}-\bar{y}\right)}{\sigma_{X} \sigma_{Y}} & \frac{\left(x_{1}-\bar{x}\right)\left(y_{2}-\bar{y}\right)}{\sigma_{X} \sigma_{Y}} & \ldots & \frac{\left(x_{1}-\bar{x}\right)\left(y_{n}-\bar{y}\right)}{\sigma_{X} \sigma_{Y}} \\
\frac{\left(x_{2}-\bar{x}\right)\left(y_{1}-\bar{y}\right)}{\sigma_{X} \sigma_{Y}} & \frac{\left(x_{2}-\bar{x}\right)\left(y_{2}-\bar{y}\right)}{\sigma_{X} \sigma_{Y}} & \ldots & \frac{\left(x_{2}-\bar{x}\right)\left(y_{n}-\bar{y}\right)}{\sigma_{X} \sigma_{Y}} \\
\vdots & \vdots & \ddots & \vdots \\
\frac{\left(x_{n}-\bar{x}\right)\left(y_{1}-\bar{y}\right)}{\sigma_{X} \sigma_{Y}} & \frac{\left(x_{n}-\bar{x}\right)\left(y_{2}-\bar{y}\right)}{\sigma_{X} \sigma_{Y}} & \ldots & \frac{\left(x_{n}-\bar{x}\right)\left(y_{n}-\bar{y}\right)}{\sigma_{X} \sigma_{Y}}
\end{array}\right] .
$$

The sum of off-diagonal elements in an $i$ th row is given by

$$
\begin{aligned}
\sum_{j \neq i} q_{i j} & =\frac{\left(x_{i}-\bar{x}\right)\left[\left(y_{1}-\bar{y}\right)+\ldots+\left(y_{i-1}-\bar{y}\right)+\left(y_{i+1}-\bar{y}\right)+\ldots+\left(y_{n}-\bar{y}\right)\right]}{\sigma_{X} \sigma_{Y}} \\
& =\frac{\left(x_{i}-\bar{x}\right)\left[\left(\sum_{i=1}^{n}\left(y_{i}-\bar{y}\right)\right)-\left(y_{i}-\bar{y}\right)\right]}{\sigma_{X} \sigma_{Y}}=\frac{-\left(x_{i}-\bar{x}\right)\left(y_{i}-\bar{y}\right)}{\sigma_{X} \sigma_{Y}} .
\end{aligned}
$$

Therefore, the sum of all the off-diagonal elements in the entire matrix $\mathbf{Q}$ is given by

$$
\begin{aligned}
\sum_{i} \sum_{j \neq i} q_{i j} & =\frac{-\sum_{i}\left(x_{i}-\bar{x}\right)\left(y_{i}-\bar{y}\right)}{\sigma_{X} \sigma_{Y}} \\
& =-n r_{X, Y} .
\end{aligned}
$$

On the other hand, the sum of all the on-diagonal elements in equation (9) is given by

$$
\begin{aligned}
\operatorname{tr}(\mathbf{Q}) & =\sum_{i} q_{i i}=\frac{\sum_{i}\left(x_{i}-\bar{x}\right)\left(y_{i}-\bar{y}\right)}{\sigma_{X} \sigma_{Y}} \\
& =n r_{X, Y} .
\end{aligned}
$$

From equations (10) and (11), it is acknowledged that the sum of off-diagonal elements is identical to Pearson's correlation coefficient between two variables, multiplied by $-n$, and that the sum of on-diagonal elements is the same as Pearson's correlation coefficient, multiplied by $n$. Hence the sum of all the elements in $\mathbf{Q}$ is equal to zero, as in $\mathbf{Q}$ for univariate measures (see table 4).

When all these definitions are applied to equation (6), one can formulate the equation for the expected value specific to each spatial association measure as seen in the last 
column in table 4 . With a binary connectivity matrix $(\mathbf{C})$ or its row-standardized version $(\mathbf{W})$, the equation reduces to the well-known equation, $-1 /(n-1)$ for Moran's $I$ and 1 for Geary's $c$, because $\operatorname{tr}(\mathbf{V})$ is equal to zero. Those familiar expected values, however, do not hold for a spatial weights matrix with nonzero diagonal elements. The equations suggested here provide for proper expected values with all types of spatial weights matrices, which will be illustrated subsequently.

The expected value for Cross - Moran is given by $-r_{X, Y} /(n-1)$ with $\mathbf{C}$ or $\mathbf{W}$, which proves Griffith's findings (Griffith, 1993, page 111; Griffith and Amrhein, 1997, page 48). The expected values for the spatial smoothing scalar $(S)$ and Lee's $L$ hardly reduce to simpler equations, because $\mathbf{V}^{\mathrm{T}} \mathbf{V}$ in both measures always has nonzero diagonal elements regardless of $\mathbf{V}$. When a row-standardized matrix $\mathbf{W}^{*}$ with nonzero diagonal elements is applied, the equations are simplified respectively to

$$
\frac{\sum_{i} \sum_{j} w_{i j}^{* 2}-1}{n-1}, \quad \text { and } \frac{\sum_{i} \sum_{j} w_{i j}^{* 2}-1}{n-1} r_{X, Y}
$$

[note that $\operatorname{tr}\left(\mathbf{V}^{\mathrm{T}} \mathbf{V}\right)=\sum_{i} \sum_{j} v_{i j}^{2}$, and $\boldsymbol{1}^{\mathrm{T}}\left(\mathbf{V}^{\mathrm{T}} \mathbf{V}\right) \boldsymbol{1}=n$ with a row-standardized spatial
weights matrix].

\section{An illustration}

As an experiment, I designed two different spatial patterns on a hypothetical space that is composed of 37 hexagons (figure 1). The two spatial patterns have the same mean (1.838) and variance (0.514) and the Pearson's correlation coefficient between them is 0.422. Pattern A is used for univariate measures, and the relation between pattern A and $\mathrm{B}$ is utilized for bivariate measures.

Four different spatial weights matrices were built to examine how the extended Mantel test works with different methods of defining spatial relationships among areal units. They are

$\mathbf{C} \equiv\left\{c_{i j}\right\}$, where $c_{i j}=1$ if $i$ and $j$ share a common boundary, and $c_{i j}=0$ otherwise; $\mathbf{W}$ : a row-standardized version of $\mathbf{C}$;

$\mathbf{C}^{*}$ : ones on the diagonal of $\mathbf{C}$;

$\mathbf{W}^{*}$ : a row-standardized version of $\mathbf{C}^{*}$.

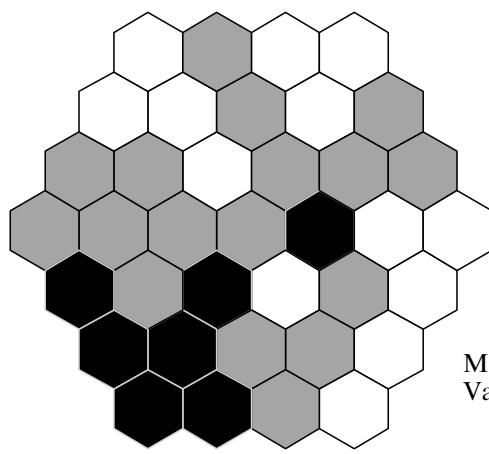

Mean: $\quad 1.838$

Variance: 0.514

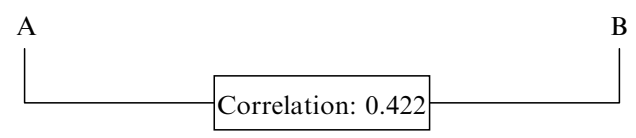

Figure 1. Hypothetical spatial patterns. 
In order to investigate how reasonable is the approximation from the extended Mantel test, I conducted a Monte Carlo simulation with 10000 permutations for each type of spatial weights matrix for each measure. Table 5 (over) lists the results along with all the elements in table 2. For all the measures over all the types of spatial weights matrices examined, the approximation drawn from the extended Mantel test appears highly reasonable for the first two moments when compared with the permutation results. This verifies that the decomposition of distributional moments into ones for off-diagonal and on-diagonal elements provides a fundamental insight into the sampling distribution of global measures of spatial association.

Overall, Moran's I and Cross - Moran appear to behave similarly. In both measures, zero-diagonal spatial weights matrices $(\mathbf{C}$ and $\mathbf{W})$ produce the same expected values whereas the matrices of $\mathbf{C}^{*}$ and $\mathbf{W}^{*}$ move the expected values to the positive side and reduce the variance to a significant extent. This is basically because of the introduction of the expected value for on-diagonal elements.

Another way to investigate the exactness of the proposed procedure on Moran's $I$ is to compare the derived moments with moments from a set of well-known equations based on the normality assumption (Anselin, 1988, page 102; Upton and Fingleton, 1985, page 338):

$$
\begin{aligned}
& \mathrm{E}(I)=\frac{n}{\mathbf{1}^{\mathrm{T}} \mathbf{V} \mathbf{1}} \frac{\operatorname{tr}(\mathbf{M V})}{n-1}, \\
& \operatorname{var}(I)=\left(\frac{n}{\mathbf{1}^{\mathrm{T}} \mathbf{V} \mathbf{1}}\right)^{2} \frac{\operatorname{tr}\left(\mathbf{M V M} \mathbf{M} \mathbf{V}^{\mathrm{T}}\right)+\operatorname{tr}\left[(\mathbf{M V})^{2}\right]+[\operatorname{tr}(\mathbf{M V})]^{2}}{(n-1)(n+1)}-[\mathrm{E}(I)]^{2},
\end{aligned}
$$

where $\mathbf{M}=\mathbf{I}_{n}-\frac{1}{n} \mathbf{1 1}^{\mathrm{T}}$. Equation (12) provides an identical set of expected values for the different spatial weights matrices, but equation (13) gives slightly different values for variance, which simply reflects the differences between the two approaches in terms of inferential assumption. The same sets of equations were derived for Geary's $c$ and the spatial smoothing scalar $(S)$ (Lee, 2001b) and the results reported the same thing.

Geary's $c$, the spatial smoothing scalar $(S)$, and Lee's $L$ have certain values in all the distributional elements in table 5, mainly because the diagonal elements of $\mathbf{P}$ are always nonzero and are usually different from one another. Here, it should be underlined that the impacts of nonzero diagonal elements on the expected value for the spatial smoothing scalar $(S)$ and Lee's $L$ are significant but are negligible on the on-diagonal variance and covariance over all the spatial weighting schemes. This suggests that the equation for off-diagonal variance alone could provide a good approximation to the overall variance and that the task of constructing equations for higher moments could be made much easier.

From the last column in table 5, one can recognize that the skewness for the measures is not negligible. Owing to the exact distribution approach (Hepple, 1998; Tiefelsdorf, 1998; 2000; Tiefelsdorf and Boots, 1995), Moran's I is no longer subject to the problem of nonnormality, and the approach could be extended for Geary's $c$ and the spatial smoothing scalar $(S)$. Unfortunately, as Tiefelsdorf (2001) shows, it is not possible to apply the exact distribution approach to bivariate spatial association measures at this point. Given the high level of positive skewness for Lee's $L$ (see also, Lee, 2001a, figure 3), a fundamental adjustment should be made for adequate inference, even though a large sample could alleviate the skewness issue to a large extent. 
Table 5. Distributional properties of five global measures of spatial association based on the extended Mantel test, compared with the results from 10000 Monte Carlo simulations.

\begin{tabular}{|c|c|c|c|c|c|c|c|c|c|c|c|c|}
\hline \multirow{2}{*}{$\begin{array}{l}\text { Spatial } \\
\text { weights } \\
\text { matrices }\end{array}$} & \multirow[t]{2}{*}{ Measure } & \multicolumn{8}{|c|}{ Extended Mantel test } & \multirow{2}{*}{\multicolumn{3}{|c|}{10000 permutations }} \\
\hline & & \multicolumn{3}{|c|}{ expected values } & \multicolumn{4}{|l|}{ variances } & $Z$ & & & \\
\hline (a) Moran's & & $\mathrm{E}(I)$ & $\mathrm{E}\left(I^{\text {off }}\right)$ & $\mathrm{E}\left(I^{\text {on }}\right)$ & $\operatorname{var}(I)$ & $\operatorname{var}\left(I^{\text {off }}\right)$ & $\operatorname{var}\left(I^{\mathrm{on}}\right)$ & $2 \operatorname{cov}\left(I^{\text {off }}, I^{\text {on }}\right)$ & $Z(I)$ & $\operatorname{mean}(I)$ & $\operatorname{var}(I)$ & skewness \\
\hline $\mathbf{C}$ & 0.3092 & -0.0278 & -0.0278 & 0 & 0.0097 & 0.0097 & 0 & 0 & 3.4240 & -0.0275 & 0.0101 & 0.3352 \\
\hline $\mathbf{W}$ & 0.3860 & -0.0278 & -0.0278 & 0 & 0.0104 & 0.0104 & 0 & 0 & 4.0621 & -0.0276 & 0.0104 & 0.3508 \\
\hline $\mathbf{C}^{*}$ & 0.4270 & 0.1475 & -0.0230 & 0.1705 & 0.0067 & 0.0067 & 0 & 0 & 3.4240 & 0.1481 & 0.0069 & 0.3401 \\
\hline $\mathbf{W}^{*}$ & 0.4919 & 0.1560 & -0.0228 & 0.1788 & 0.0069 & 0.0068 & $4.35 \times 10^{-5}$ & $1.23 \times 10^{-5}$ & 4.0397 & 0.1555 & 0.0068 & 0.3719 \\
\hline \multicolumn{2}{|c|}{ (b) Geary's c } & $\mathrm{E}(c)$ & $\mathrm{E}\left(c^{\text {off }}\right)$ & $\mathrm{E}\left(c^{\text {on }}\right)$ & $\operatorname{var}(c)$ & $\operatorname{var}\left(c^{\text {off }}\right)$ & $\operatorname{var}\left(c^{\mathrm{on}}\right)$ & $2 \operatorname{cov}\left(c^{\text {off }}, c^{\text {on }}\right)$ & $Z(c)$ & $\operatorname{mean}(c)$ & $\operatorname{var}(c)$ & skewness \\
\hline $\mathbf{C}$ & 0.6202 & 1 & 0.0270 & 0.9730 & 0.0109 & 0.0092 & 0.0016 & 0.0002 & -3.6350 & 1.0000 & 0.0108 & 0.2000 \\
\hline W & 0.5774 & 1 & 0.0270 & 0.9730 & 0.0101 & 0.0098 & 0.0003 & $3.09 \times 10^{-5}$ & -4.1998 & 1.0005 & 0.0101 & 0.3609 \\
\hline $\mathbf{C}^{*}$ & 0.5144 & 0.8295 & 0.0224 & 0.8071 & 0.0075 & 0.0063 & 0.0011 & 0.0001 & -3.6350 & 0.8289 & 0.0077 & 0.2138 \\
\hline $\mathbf{W}^{*}$ & 0.4810 & 0.8212 & 0.0222 & 0.7990 & 0.0068 & 0.0065 & 0.0003 & $3.18 \times 10^{-5}$ & -4.1241 & 0.8213 & 0.0068 & 0.3474 \\
\hline \multicolumn{2}{|c|}{$\begin{array}{l}\text { (c) Lee's Spatial } \\
\text { Smoothing Scalar (S) }\end{array}$} & $\mathrm{E}(S)$ & $\mathrm{E}\left(S^{\mathrm{off}}\right)$ & $\mathrm{E}\left(S^{\mathrm{on}}\right)$ & $\operatorname{var}(S)$ & $\operatorname{var}\left(S^{\text {off }}\right)$ & $\operatorname{var}\left(S^{\text {on }}\right)$ & $2 \operatorname{cov}\left(S^{\text {off }}, S^{\text {on }}\right)$ & $Z(S)$ & $\operatorname{mean}(S)$ & $\operatorname{var}(S)$ & skewness \\
\hline C & 0.3489 & 0.1711 & -0.0224 & 0.1935 & 0.0019 & 0.0019 & $6.20 \times 10^{-5}$ & $-3.83 \times 10^{-5}$ & 4.0351 & 0.1715 & 0.0020 & 0.8275 \\
\hline W & 0.4177 & 0.1991 & -0.0216 & 0.2207 & 0.0025 & 0.0025 & $7.86 \times 10^{-5}$ & $-1.91 \times 10^{-5}$ & 4.3547 & 0.1988 & 0.0025 & 0.7252 \\
\hline $\mathbf{C}^{*}$ & 0.3563 & 0.1403 & -0.0232 & 0.1635 & 0.0026 & 0.0026 & $3.05 \times 10^{-5}$ & $-2.58 \times 10^{-5}$ & 4.2176 & 0.1403 & 0.0026 & 0.8980 \\
\hline $\mathbf{W}^{*}$ & 0.4361 & 0.1560 & -0.0228 & 0.1788 & 0.0031 & 0.0031 & $1.67 \times 10^{-5}$ & $-4.58 \times 10^{-6}$ & 5.0374 & 0.1565 & 0.0031 & 0.8567 \\
\hline \multicolumn{2}{|c|}{$\begin{array}{l}\text { (d) Wartenberg's } \\
\text { Cross - Moran (CM) }\end{array}$} & $\mathrm{E}(C M)$ & $\mathrm{E}\left(C M^{\text {off }}\right)$ & $\mathrm{E}\left(C M^{\mathrm{on}}\right)$ & $\operatorname{var}(C M)$ & $\operatorname{var}\left(C M^{\text {off }}\right)$ & $\operatorname{var}\left(C M^{\mathrm{on}}\right)$ & $2 \operatorname{cov}\left(C M^{\text {off }}, C M^{\text {on }}\right)$ & $Z(C M)$ & $\operatorname{mean}(C M)$ & $\operatorname{var}(C M)$ & skewness \\
\hline $\begin{array}{l}\text { Cross }- \text { Mo } \\
\text { C }\end{array}$ & $\begin{array}{l}\operatorname{ran}(\mathrm{CM}) \\
0.3358\end{array}$ & -0.0117 & -0.0117 & 0 & 0.0057 & 0.0057 & 0 & 0 & 4.5896 & -0.0113 & 0.0057 & 0.2492 \\
\hline W & 0.3572 & -0.0117 & -0.0117 & 0 & 0.0061 & 0.0061 & 0 & 0 & 4.7088 & -0.0123 & 0.0063 & 0.2586 \\
\hline $\mathbf{C}^{*}$ & 0.3505 & 0.0622 & -0.0097 & 0.0719 & 0.0039 & 0.0039 & 0 & 0 & 4.5896 & 0.0628 & 0.0041 & 0.2513 \\
\hline $\mathbf{W}^{*}$ & 0.3688 & 0.0658 & -0.0096 & 0.0754 & 0.0041 & 0.0041 & $3.98 \times 10^{-5}$ & $1.12 \times 10^{-5}$ & 4.7266 & 0.0667 & 0.0042 & 0.2341 \\
\hline \multicolumn{2}{|l|}{ (e) Lee's $L$} & $\mathrm{E}(L)$ & $\mathrm{E}\left(L^{\text {off }}\right)$ & $\mathrm{E}\left(L^{\text {on }}\right)$ & $\operatorname{var}(L)$ & $\operatorname{var}\left(L^{\text {off }}\right)$ & $\operatorname{var}\left(L^{\text {on }}\right)$ & $2 \operatorname{cov}\left(L^{\text {off }}, L^{\text {on }}\right)$ & $Z(L)$ & $\operatorname{mean}(L)$ & $\operatorname{var}(L)$ & skewness \\
\hline C & 0.2769 & 0.0722 & -0.0095 & 0.0817 & 0.0012 & 0.0011 & $5.66 \times 10^{-5}$ & $-3.50 \times 10^{-5}$ & 6.0157 & 0.0719 & 0.0012 & 0.6053 \\
\hline W & 0.3269 & 0.0840 & -0.0091 & 0.0931 & 0.0015 & 0.0015 & $7.17 \times 10^{-5}$ & $-1.74 \times 10^{-5}$ & 6.2487 & 0.0836 & 0.0015 & 0.5488 \\
\hline $\mathbf{C}^{*}$ & 0.2969 & 0.0592 & -0.0098 & 0.0690 & 0.0016 & 0.0016 & $2.78 \times 10^{-5}$ & $-2.35 \times 10^{-5}$ & 6.0284 & 0.0591 & 0.0015 & 0.6901 \\
\hline $\mathbf{W}^{*}$ & 0.3346 & 0.0658 & -0.0096 & 0.0754 & 0.0018 & 0.0018 & $1.53 \times 10^{-5}$ & $-4.18 \times 10^{-6}$ & 6.2757 & 0.0654 & 0.0019 & 0.5982 \\
\hline
\end{tabular}

weights matrices expected values ariances 


\section{Concluding remarks}

This paper has shown that spatial association measures can be presented in accordance with Mantel's generalized cross-product statistic by adequately defining matrices $\mathbf{P}$ and Q. It also demonstrates that the proposed extended Mantel test can be applied to all the measures under investigation, univariate or bivariate, over all the different forms of spatial weights matrices, zero or nonzero diagonals. Most importantly, this successfully provides a set of equations of the expected value and variance for a new bivariate spatial association measure, Lee's $L$. Furthermore, the method presented here could offer a solid inferential foundation for new spatial association measures.

Given a high level of skewness and the impossibility of pursuing the exact distribution approach, a further step should be taken for Lee's $L$. This could become more serious when it is acknowledged that the distribution of Mantel's cross-product statistic is not asymptotically normal (Mielke, 1979; Siemiatycki, 1978). This issue of nonnormality is also associated with the impacts of geometrical characteristics of spatial units on distributional properties and thus with the validity of the assumption of the asymptotic normality. As demonstrated by Boots and Tiefelsdorf (2000), a tessellation of hexagons has a much shorter feasible range of Moran's $I$ in the negative direction than tessellations of squares and triangles. More importantly, even though the validity of the asymptotic normality assumption becomes more sustainable as the number of observations increases (say, a sample size more than 50), this property is less operative for hexagons than for other tessellations, and may require a sample size of more than 100 (page 329). Highly positive skewness values for all the measures listed in the last column in table 5 are obviously associated with the fact that the spatial configuration in this paper is based on hexagons and the sample size is rather small $(n=37)$. Furthermore, extremely high skewness values for Lee's $S$ and $L$ imply that the validity of the asymptotic normality assumption and thus normal approximation for the measures would be much less reliable than any other measure even with a large sample size.

A simple way to deal with this nonnormality problem is to take some transformations with the expected value and variance (Heo and Gabriel, 1998). An initial step for a more comprehensive solution, however, is to compute higher moments for skewness and kurtosis as presented (Hubert, 1987; Mielke, 1979; Siemiatycki, 1978). Because table 5 reports that the elements of off-diagonal variance and covariance are negligible for Lee's $S$ and $L$, the procedures for deriving higher moments could be applied to the measures without being bothered by nonzero diagonals, which will be attempted elsewhere. When skewness is approximated, a Pearson Type III (gamma) function could be applied to take skewness into account for a more reliable inferential test (Costanzo et al, 1983). When both skewness and kurtosis are known, they can be utilized to fit a beta distribution (Hepple, 1998).

The extended Mantel test presented here could also offer a valuable insight into investigating distributional behaviors of local measures of spatial association, not only univariate LISA (for example, Anselin, 1995; Bao and Henry, 1996; Getis and Ord, 1996; Leung et al, 2003; Ord and Getis, 2001; Sokal et al, 1998; Tiefelsdorf, 1998; 2002) but also bivariate counterparts such as local Cross-Moran and local $L_{i}$ (Lee, 2001a; 2001b; 2003). As Tiefelsdorf demonstrates (1998; 2000), a local measure of spatial association can be obtained and tested by applying a star-shaped local spatial weights matrix for each areal unit to the corresponding global spatial association measure and significance testing. This implies that the extended Mantel test can be applied to local measures by replacing the global matrix $\mathbf{P}$ with its local version of $\mathbf{P}_{i}$, which will be examined elsewhere. 
Acknowledgements. I wish to thank the anonymous reviewers for their invaluable comments. I am tremendously indebted to Dr Michael Tiefelsdorf at the Ohio State University for developing the main ideas presented in this paper.

\section{References}

Anselin L, 1988 Spatial Econometrics: Methods and Models (Kluwer Academic, Boston, MA)

Anselin L, 1995, "Local indicators of spatial association: LISA" Geographical Analysis 27 93-115

Anselin L, Rey S, 1991, "Properties of tests for spatial dependence in linear-regression models" Geographical Analysis 23112 - 131

Bao S, Henry M, 1996, "Heterogeneity issues in local measurements of spatial association" Geographical Systems 31 - 13

Boots B N, Tiefelsdorf M, 2000, "Global and local spatial autocorrelation in bounded regular tessellations" Journal of Geographical Systems $2319-348$

Brunsdon C, Fotheringham A S, Charlton M, 2002, "Geographically weighted summary statistics - a framework for localised exploratory data analysis" Computers, Environment and Urban Systems $26501-524$

Cliff A D, Ord J K, 1981 Spatial Processes: Models and Applications (Pion, London)

Costanzo M, Hubert L J, Golledge RG, 1983, "A higher moment for spatial statistics" Geographical Analysis $15347-351$

Fotheringham A S, Brunsdon C, Charlton M, 2002 Geographically Weighted Regression: The Analysis of Spatially Varying Relationships (John Wiley, Chichester, Sussex)

Getis A, 1991, "Spatial interaction and spatial autocorrelation: a cross product approach" Environment and Planning A $231269-1277$

Getis A, Ord J K, 1996, "Local spatial statistics: an overview", in Spatial Analysis: Modelling in a GIS Environment Eds P Longley, M Batty (GeoInformation International, Cambridge) pp $261-277$

Glick B J, 1979, “Tests for space-time clustering used in cancer research" Geographical Analysis $11202-208$

Goodchild M F, 1986 Spatial Autocorrelation. Concepts and Techniques in Modern Geography 47 (Geo Books, Norwich)

Griffith D A, 1987 Spatial Autocorrelation: A Primer (Association of American Geographers, Washington, DC)

Griffith D A, 1993, "Which spatial statistics techniques should be converted to GIS functions?", in Geographic Information Systems, Spatial Modelling and Policy Evaluation Eds M Fischer, P Nijkamp (Springer, Berlin) pp $101-114$

Griffith D A, 1995, "The general linear model and spatial autoregressive models", in New Directions in Spatial Econometrics Eds L Anselin, R J G M Florax (Springer, Berlin) pp 273-295

Griffith D A, Amrhein C G, 1997 Multivariate Statistical Analysis for Geographers (Prentice-Hall, Upper Saddle River, NJ)

Griffith D A, Layne L J, 1999 A Casebook for Spatial Statistical Data Analysis: A Compilation of Analyses of Different Thematic Data Sets (Oxford University Press, New York)

Haining R, 1990 Spatial Data Analysis in the Social and Environmental Sciences (Cambridge University Press, New York)

Haining R, 2003 Spatial Data Analysis: Theory and Practice (Cambridge University Press, New York)

Heo M, Gabriel K R, 1998, "A permutation test of association between configurations by means of the RV coefficient" Communications in Statistics: Simulation and Computation 27 843-856

Hepple L W, 1998, "Exact testing for spatial autocorrelation among regression residuals" Environment and Planning A 30 85-108

Hubert L J, 1978, "Nonparametric tests for patterns in geographic variation: possible generalizations" Geographical Analysis $1086-88$

Hubert L J, 1987 Assignment Methods in Combinatorial Data Analysis (Marcel Dekker, New York)

Hubert L J, Golledge R G, 1982, "Measuring association between spatially defined variables: Tøstheim's index and some extensions" Geographical Analysis 14 273-278

Hubert L J, Golledge R G, Costanzo C M, 1981, "Generalized procedures for evaluating spatial autocorrelation" Geographical Analysis $13273-278$

Hubert L J, Golledge R G, Costanzo C M, Gale N, 1985, "Measuring association between spatially defined variables: an alternative procedure" Geographical Analysis 1736 - 46

Kelejian H H, Robinson D P, 1998, "A suggested test for spatial autocorrelation and/or heteroskedasticity and corresponding Monte Carlo results" Regional Science and Urban Economics $28389-417$ 
Lee S-I, 2001a, "Developing a bivariate spatial association measure: an integration of Pearson's $r$ and Moran's I" Journal of Geographical Systems 3369 - 385

Lee S-I, 2001b Spatial Association Measures for an ESDA - GIS Framework: Developments, Significance Tests, and Applications to Spatio-temporal Income Dynamics of U.S. Labor Market Areas, 1969 - 1999 unpublished PhD dissertation, Department of Geography, The Ohio State University, Columbus, $\mathrm{OH}$

Lee S-I, 2004, "Exploring bivariate spatial dependence and heterogeneity: a local bivariate spatial association measure for exploratory spatial data analysis (ESDA)" International Journal of Geographical Information Science (in revision)

Leung Y, Mei C-L, Zhang W-X, 2003, "Statistical test for local patterns of spatial association" Environment and Planning A $35725-744$

Mantel N, 1967, "The detection of disease clustering and a generalized regression approach" Cancer Research $27209-220$

Mantel N, Valand R S, 1970, "A technique of nonparametric multivariate analysis" Biometrics 26 $547-558$

Mielke P W, 1979, "On asymptotic non-normality of null distributions of MRPP statistics" Communications in Statistics: Theory and Methods A8 1541 - 1550; 1981, erratum A10 1795; 1982, erratum A 11847

Odland J, 1988 Spatial Autocorrelation (Sage, Newbury Park, CA)

Ord J K, Getis A, 2001, "Testing for local spatial autocorrelation in the presence of global autocorrelation" Journal of Regional Science 41411 - 432

Siemiatycki J, 1978, "Mantel's space - time clustering statistic: computing higher moments and a composition of various data transforms" Journal of Statistical Computation and Simulation 7 $13-31$

Sokal R R, 1979, “Testing statistical significance of geographical variation patterns” Systematic Zoology $28227-232$

Sokal R R, Oden N L, Thomson B A, 1998, "Local spatial autocorrelation in a biological model" Geographical Analysis 30331 - 354

Tiefelsdorf M, 1998, "Some practical applications of Moran's I's exact conditional distribution" Papers in Regional Science 77101 - 129

Tiefelsdorf M, 2000 Modelling Spatial Processes: The Identification and Analysis of Spatial Relationships in Regression Residuals by Means of Moran's I (Springer, Berlin)

Tiefelsdorf M, 2001, "Specification and distributional properties of the spatial cross-correlation coefficient $C_{\varepsilon_{1}, \varepsilon_{2}}$ ", paper presented at the Western Regional Science Conference, Palm Springs, 26 February; copy available from the author, Department of Geography, Ohio State University, Columbus, $\mathrm{OH}$

Tiefelsdorf M, 2002, "The saddlepoint approximation of Moran's $I$ 's and local Moran's $I_{i}$ 's reference distributions and their numerical evaluation" Geographical Analysis 34 187-206

Tiefelsdorf M, Boots B, 1995, "The exact distribution of Moran's I" Environment and Planning A $27985-999$

Upton G J G, Fingleton B, 1985 Spatial Data Analysis by Example. Volume 1: Point Pattern and Quantitative Data (John Wiley, New York)

Wartenberg D, 1985, "Multivariate spatial correlation: a method for exploratory geographical analysis" Geographical Analysis 17263 - 283 


\section{Appendix}

\section{Original equations for the five elements in table 2}

The expected value for off-diagonal elements (Cliff and Ord, 1981; Mantel, 1967) is given by

$$
\begin{aligned}
\mathrm{E}\left(\Gamma^{\mathrm{off}}\right) & =\mathrm{E}\left(\sum_{i} \sum_{j \neq i} p_{i j} q_{i j}\right) \\
& =\sum_{i} \sum_{j \neq i} p_{i j} \mathrm{E}\left(q_{i j}\right)=\sum_{i} \sum_{j \neq i} p_{i j} \frac{\sum_{i} \sum_{j \neq i} q_{i i}}{n(n-1)} .
\end{aligned}
$$

The expected value for on-diagonal elements can be given (Heo and Gabriel, 1998, page 848):

$$
\begin{aligned}
\mathrm{E}\left(\Gamma^{\mathrm{on}}\right) & =\mathrm{E}\left(\sum_{i} p_{i i} q_{i i}\right) \\
& =\sum_{i} p_{i i} \mathrm{E}\left(q_{i i}\right)=\sum_{i} p_{i i} \frac{\sum_{i} q_{i i}}{n} .
\end{aligned}
$$

The variance for off-diagonal elements (Cliff and Ord, 1981; Mantel, 1967) is defined as

$$
\begin{aligned}
& \operatorname{var}\left(\Gamma^{\mathrm{off}}\right)= \operatorname{var}\left(\sum_{i} \sum_{j \neq i} p_{i j} q_{i j}\right) \\
&= \sum_{i} \sum_{j \neq i} \sum_{k} \sum_{l \neq k} p_{i j} p_{k l} \operatorname{cov}\left(q_{i j}, q_{k l}\right) \\
&= \frac{2 \sum_{i} \sum_{j \neq i} p_{i j}^{2} \sum_{i} \sum_{j \neq i} q_{i j}^{2}}{n(n-1)} \\
&+\left\{\frac{4\left[\sum_{i}\left(\sum_{j \neq i} p_{i j}\right)^{2}-\sum_{i} \sum_{j \neq i} p_{i j}^{2}\right]\left[\sum_{i}\left(\sum_{j \neq i} q_{i j}\right)^{2}-\sum_{i} \sum_{j \neq i} q_{i j}^{2}\right]}{n(n-1)(n-2)(n-3)}\left[\left(\sum_{i} \sum_{j \neq i} p_{i j}\right)^{2}+2 \sum_{i} \sum_{j \neq i} p_{i j}^{2}-4 \sum_{i}\left(\sum_{j \neq i} p_{i j}\right)^{2}\right]\right. \\
&\left.\times\left[\left(\sum_{i} \sum_{j \neq i} q_{i j}\right)^{2}+2 \sum_{i} \sum_{j \neq i} q_{i j}^{2}-4 \sum_{i}\left(\sum_{j \neq i} q_{i j}\right)^{2}\right]\right\}-\left[\frac{\sum_{i} \sum_{j \neq i} p_{i j} \sum_{i} \sum_{j \neq i} q_{i j}}{n(n-1)}\right]^{2} .
\end{aligned}
$$


The variance for on-diagonal elements (Heo and Gabriel, 1998, page 848) is given accordingly:

$$
\begin{aligned}
\operatorname{var}\left(\Gamma^{\mathrm{on}}\right)= & \operatorname{var}\left(\sum_{i} p_{i i} q_{i i}\right) \\
= & \sum_{i} \sum_{j} p_{i i} p_{j j} \operatorname{cov}\left(q_{i i}, q_{j j}\right) \\
= & \frac{\sum_{i} p_{i i}^{2} \sum_{i} q_{i i}^{2}}{n}+\frac{\sum_{i} \sum_{j \neq i} p_{i i} p_{j j} \sum_{i} \sum_{j \neq i} q_{i i} q_{j j}}{n(n-1)}-\left(\frac{\sum_{i} p_{i i} \sum_{i} q_{i i}}{n}\right)^{2} \\
= & \frac{\sum_{i} p_{i i}^{2} \sum_{i} q_{i i}^{2}}{n}+\frac{\left[\left(\sum_{i} p_{i i}\right)^{2}-\sum_{i} p_{i i}^{2}\right]\left[\left(\sum_{i} q_{i i}\right)^{2}-\sum_{i} q_{i i}^{2}\right]}{n(n-1)} \\
& -\left(\frac{\sum_{i} p_{i i} \sum_{i} q_{i i}}{n}\right)^{2} .
\end{aligned}
$$

Finally, the covariance between off-diagonal and on-diagonal elements (Heo and Gabriel, 1998, page 848) is given by

$$
\begin{aligned}
\operatorname{cov}\left(\Gamma^{\mathrm{off}}, \Gamma^{\mathrm{on}}\right)= & \sum_{i} \sum_{j} \sum_{k \neq j} p_{i i} p_{j k} \operatorname{cov}\left(q_{i i}, q_{j k}\right) \\
= & \frac{2 \sum_{i} \sum_{j \neq i} p_{i i} p_{i j} \sum_{i} \sum_{j \neq i} q_{i i} q_{i j}}{n(n-1)} \\
& +\frac{\sum_{i} \sum_{j \neq i} \sum_{k \neq j} p_{i i} p_{j k} \sum_{i} \sum_{j \neq i} \sum_{k \neq j} q_{i i} q_{j k}}{n(n-1)(n-2)} \\
& -\frac{\sum_{i} \sum_{j \neq i} p_{i j} \sum_{i} \sum_{j \neq i} q_{i j}}{n(n-1)} \frac{\sum_{i} p_{i i} \sum_{i} q_{i i}}{n} .
\end{aligned}
$$


